\title{
Correction to: Occupational exposure to electromagnetic fields in magnetic resonance environment: an update on regulation, exposure assessment techniques, health risk evaluation, and surveillance
}

\author{
Valentina Hartwig ${ }^{1}$ (D) Giorgio Virgili ${ }^{1} \cdot$ Federica Mattei $^{2} \cdot$ Cristiano Biagini $^{3} \cdot$ Stefania Romeo $^{4} \cdot$ Olga Zeni $^{5}$. \\ Maria Rosaria Scarfi ${ }^{5} \cdot$ Rita Massa $^{5} \cdot$ Francesco Campanella $^{5,6} \cdot$ Luigi Landini $^{7} \cdot$ Fabriziomaria Gobba $^{8}$. \\ Alberto Modenese ${ }^{9} \cdot$ Giulio Giovannetti ${ }^{9}$
}

Published online: 13 January 2022

(c) International Federation for Medical and Biological Engineering 2022

\section{Correction to: Medical \& Biological Engineering \& Computing} https://doi.org/10.1007/s11517-021-02435-6

The original article contained a mistake.

The original article reports wrong affiliations.

The final affiliations have reported below:

Valentina Hartwig1 · Giorgio Virgili2 · Federica Mattei3 . Cristiano Biagini4 · Stefania Romeo5 · Olga Zeni5

Maria Rosaria Scarfí · Rita Massa5,6 · Francesco Campanella7 . Luigi Landini8 · Fabriziomaria Gobba9 · Alberto Modenese9 . Giulio Giovannetti1

The original article can be found online at https://doi.org/10.1007/ s11517-021-02435-6.

Valentina Hartwig

valeh@ifc.cnr.it

1 Institute of Clinical Physiology (IFC), Italian National Research Council (CNR), Via G. Moruzzi 1, 56124 Pisa, San Cataldo, Italy

2 Virgili Giorgio, Via G. Pastore 2, 26040 Crespina-Lorenzana, Italy

3 West Systems S.R.L, Via Don Mazzolari 25, 56025 Pontedera, PI, Italy

4 Associazione Italiana Tecnici Dell'Imaging in Risonanza Magnetica, AITIRM, Via XX Settembre 76, 50129 Florence, Italy

5 Institute for Electromagnetic Sensing of the Environment (IREA), Italian National Research Council (CNR), Via Diocleziano 328, 80124 Naples, Italy
1 Institute of Clinical Physiology (IFC), Italian National Research Council (CNR), Via G. Moruzzi 1, 56124 Pisa, San Cataldo, Italy

2 Virgili Giorgio, Via G. Pastore 2, 26040 Crespina-Lorenzana, Italy

3 West Systems S.R.L, Via Don Mazzolari 25, 56025 Pontedera, PI, Italy
6 Department of Physics 'Ettore Pancini', University of Naples Federico II, Via Cinthia 21, 80126 Naples, Italy

7 Dipartimento di Medicina, Epidemiologia, Igiene del Lavoro e Ambientale, Inail, Via Fontana Candida 1, Rome 00078 Monte Porzio Catone, Italy

8 Fondazione Toscana Gabriele Monasterio, Via G. Moruzzi 1, 56124 Pisa, San Cataldo, Italy

9 Department of Biomedical, Metabolic and Neural Sciences, University of Modena and Reggio Emilia, Via Campi 287, 41125 Modena, Italy 
4 Associazione Italiana Tecnici Dell'Imaging in Risonanza Magnetica, AITIRM, Via XX Settembre 76, 50129 Florence, Italy

5 Institute for Electromagnetic Sensing of the Environment (IREA), Italian National Research Council (CNR), Via Diocleziano 328, 80124 Naples, Italy

6 Department of Physics 'Ettore Pancini', University of Naples Federico II, Via Cinthia 21, 80126 Naples, Italy

7 Dipartimento di medicina, epidemiologia, igiene del lavoro e ambientale, Inail, Via Fontana Candida 1, 00078 Monte Porzio Catone, Rome, Italy
8 Fondazione Toscana Gabriele Monasterio, Via G. Moruzzi 1, 56124 Pisa, San Cataldo, Italy

9 Department of Biomedical, Metabolic and Neural Sciences, University of Modena and Reggio Emilia, Via Campi 287, 41125 Modena, Italy

The original article has been corrected.

Publisher's note Springer Nature remains neutral with regard to jurisdictional claims in published maps and institutional affiliations. 